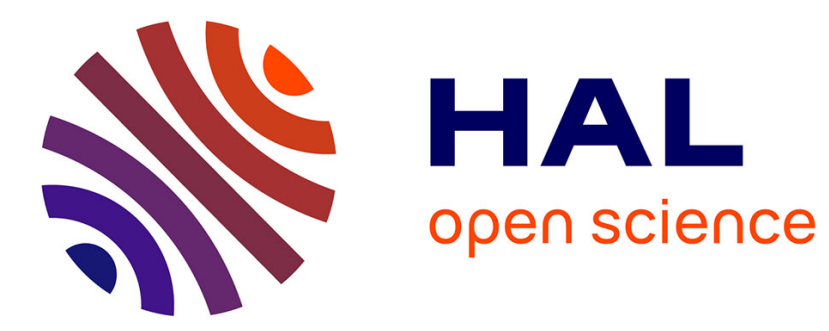

\title{
In-phase and antiphase self-intensity regulated dual-frequency laser using two-photon absorption
}

Abdelkrim El Amili, Kevin Audo, Mehdi Alouini

\section{To cite this version:}

Abdelkrim El Amili, Kevin Audo, Mehdi Alouini. In-phase and antiphase self-intensity regulated dual-frequency laser using two-photon absorption. Optics Letters, 2016, 41 (10), pp.2326-2329. 10.1364/OL.41.002326 . hal-01343404

\section{HAL Id: hal-01343404 https://hal-univ-rennes1.archives-ouvertes.fr/hal-01343404}

Submitted on 23 Feb 2017

HAL is a multi-disciplinary open access archive for the deposit and dissemination of scientific research documents, whether they are published or not. The documents may come from teaching and research institutions in France or abroad, or from public or private research centers.
L'archive ouverte pluridisciplinaire HAL, est destinée au dépôt et à la diffusion de documents scientifiques de niveau recherche, publiés ou non, émanant des établissements d'enseignement et de recherche français ou étrangers, des laboratoires publics ou privés. 


\title{
In-phase and anti-phase self-intensity regulated dual frequency laser using two-photon absorption
}

\author{
Abdelkrim El Amili, ${ }^{1}$ KeVin Audo, ${ }^{1}$ Mehdi Alouini ${ }^{1, *}$ \\ ${ }^{1}$ Institut de Physique de Rennes, Université de Rennes 1, CNRS, Campus de Beaulieu, 35042 Rennes, France \\ *Corresponding author: mehdi.alouini@univ-rennes1.fr
}

\begin{abstract}
A $25 \mathrm{~dB}$ reduction of resonant intensity noise spectra is experimentally demonstrated for both the anti-phase and in-phase relaxation oscillations of a dual-frequency solid-state laser operating at the telecommunication wavelengths. Experimental results demonstrate that incorporation of an intra-cavity two-photon absorber that acts as a buffer reservoir reduces efficiently the inphase noise contribution, while it is somewhat ineffective in lowering the anti-phase noise contributions. A slight spatial separation of the two modes in the nonlinear twophoton absorber reduces the anti-phase resonant intensity noise component. These experimental results provide a new approach in the design of ultra-low noise dual-frequency solid state lasers. (C) 2015 Optical Society of America
\end{abstract}

OCIS codes: (140.3480) Lasers, diode-pumped, (140.3580) Lasers, solidstate, (270.2500) Fluctuations, relaxations, relative intensity noise, and Two-photon absorption.

http://dx.doi.org/10.1364/OL.99.099999

Dual-frequency solid-state lasers are of great interest for many practical applications, such as metrology [1, 2], lidar-radar [3], wireless communications [4] and microwave photonics [5-7]. In particular, the dual-frequency lasers, whose their two modes are cross-polarized, offer tunability of the frequency difference with an inherently narrow linewidth of the beatnote. Moreover, the solidstate lasers are also known for their intrinsic low-phase noise and high optical power. Unfortunately, they suffer from resonant noise peaks at the relaxation oscillation (RO) frequency. Particularly, the dual-frequency solid-state lasers that support two laser modes, exhibit resonant noise at two eigen-frequencies associated with the so-called anti-phase and in-phase noise spectra. The in-phase noise corresponding to the common RO results from the nonlinear interaction between the population inversion and the total intracavity photon population. The RO mechanism is inherently present in any laser that the population inversion lifetime is longer than the cavity photon lifetime and are classified as class B lasers [8,9]. The anti-phase noise dominates at lower frequencies and follows the coupling dynamics of the two laser modes through nonlinear gain saturation of the active gain medium $[10,11]$. Its dynamics leads to a damped resonant exchange of energy between the two laser modes (cf. Fig. 2). The presence of this anti-phase noise is undoubtedly a major drawback of dual-polarized solid state lasers since its resonance suppression mechanism is not so straightforward contrary to the RO noise suppression. It therefore limits utility of the orthogonally polarized lasing modes in applications where a low intensity noise levels are required for the generated microwave beat note.

Therefore, different methods have been reported to reduce either the anti-phase noise or the in-phase noise spectra; the antiphase noise can be reduced by designing a two axis laser topology, where the two modes are spatially separated in the active medium, leading to the cancellation of their coupling mechanism [12-14]. Another approach consists of an active medium with the appropriate crystal cut in conjunction with a proper orientation of the two eigen-polarizations [15]. Moreover, the in-phase intensity noise can be dampened either electronically or optically using feedback loop mechanisms [16, 17]. An attractive alternative solution is based on dramatic modification of the laser dynamics by establishing a class-A laser [18], where the population inversion lifetime is shorter than the cavity photon lifetime. This approach is a viable solution only for the high finesse external cavity based semiconductor lasers [19].

Recently a promising approach has been explored for the solid state lasers by incorporating a buffer reservoir (BR) into the laser cavity, where the exclusive interaction between the population inversion and the photon population [20-22] are restricted. This approach has been experimentally validated in the single frequency lasers, but never in dual frequency solid state lasers. More precisely, one can explore whether the incorporation of such a BR in a dual frequency lasers can reduce contribution of the inphase and anti-phase noise dynamics.

This letter presents the incorporation of BR approach in a dual frequency solid-state laser system. In addition, we also propose for the first time a new laser cavity design to get rid of both in-phase and anti-phase noise sources. 
The laser system used for this investigation (shown in Fig. 1(a)) is based on a 4.9-cm-long planar-concave cavity. The active medium is a 1.5-mm-long phosphate glass co-doped with Erbium and Ytterbium (Er,Yb:glass). The first side of the Er,Yb:glass plate, which acts as the cavity input mirror, is coated for high reflectivity at $1550 \mathrm{~nm}(\mathrm{R}>99.9 \%)$ and high transmission $(\mathrm{T}=95 \%)$ at the pump wavelength $(975 \mathrm{~nm}$ ). The cavity is closed by a 5 -cm-radius of curvature mirror transmitting $0.5 \%$ of the intensity at $1560 \mathrm{~nm}$. The gain medium is pumped by a multimode fiber-coupled semiconductor laser diode operating at $975 \mathrm{~nm}$. An AR-coated $200-\mu \mathrm{m}$-thick birefringent $\mathrm{YVO}_{4}$ crystal, cut at $45^{\circ}$ with respect to its optical axis, is inserted into the laser cavity in order to slightly reduce the coupling strength between the two polarization modes and thus increase the robustness of dual mode operation. This birefringent crystal basically introduces a spatial separation of 20 $\mu \mathrm{m}$ between the two orthogonal modes in the active medium. This separation is very small compared to the $\sim 120 \mu \mathrm{m}$ of beam diameter and thus considered as a single axis dual-frequency laser and can consequently be pumped with a single pump spot. The two modes are continuously monitored with a Fabry-Perot cavity to check that the laser remains single mode without any mode hopping during data acquisition. The laser output is analyzed using a 3.7-MHz-bandwidth photodiode. A half-wave plate (HWP) followed by an optical isolator is inserted in front of the photodiode. By rotating the HWP one can project the laser output on any linear polarization state so that the two laser eigenpolarizations can be analyzed independently or combined. The noise spectra are finally recorded using an electrical spectrum analyzer (ESA) from Rohde \& Schwarz (model FSV 10 Hz - 3.6 $\mathrm{GHz}$ ).
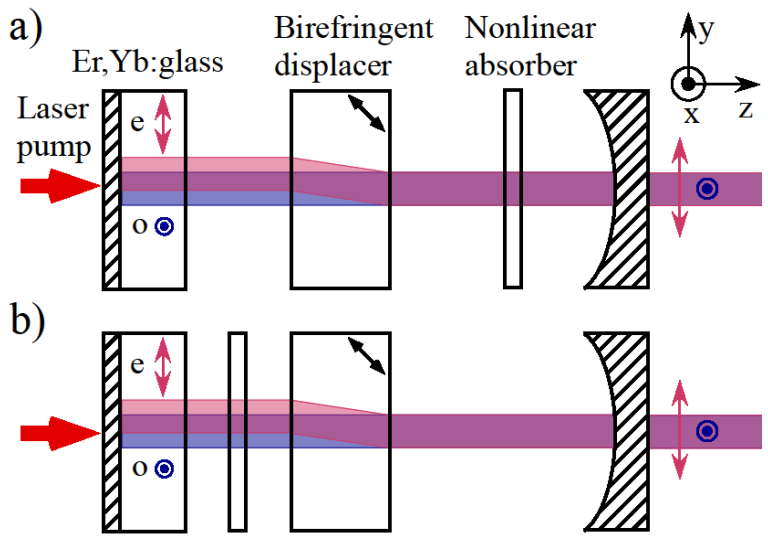

Fig. 1. Schematic representation of the two studied laser architectures. The birefringent displacer is an $\mathrm{YVO}_{4}$ crystal cut at $45^{\circ}$ from it optical axis that induces a $20 \mu \mathrm{m}$ spatial separation between the two orthogonally polarized modes. A $100-\mu$ m-thick Silicon crystal is used as the nonlinear absorber. (a) The two polarized modes are slightly separated only in the active medium. (b) The two polarized modes are slightly separated in both the active medium and the nonlinear absorber.

To conduct our study a silica etalon is added to the standard solid state laser structure to induce single longitudinal mode oscillation of the two polarization states. This etalon also being used as the BR to control RIN spectra in this study. Fig. 2 depicts a typical recorded relative intensity noise (RIN) spectra, when the optical amplitudes of both modes are carefully equalized. The RIN spectra labelled (1) and (2) correspond respectively to that of the $\mathrm{x}$ - and $\mathrm{y}$-polarized modes, where they exhibit a strong peak at around $20 \mathrm{kHz}$ corresponding to the anti-phase resonant noise and a second peak at around $45 \mathrm{kHz}$ corresponding to the in-phase resonant noise. The presence of the strong anti-phase peak proves that the spatial separation between the two modes is so weak that the two modes remain highly coupled in the active medium. This is confirmed by rotating the HWP in front of the polarizing isolator by $22.5^{\circ}$. In this case, the optical amplitudes of the two modes are constructively added and the anti-phase peak cancels out, while the in-phase peak remains present.

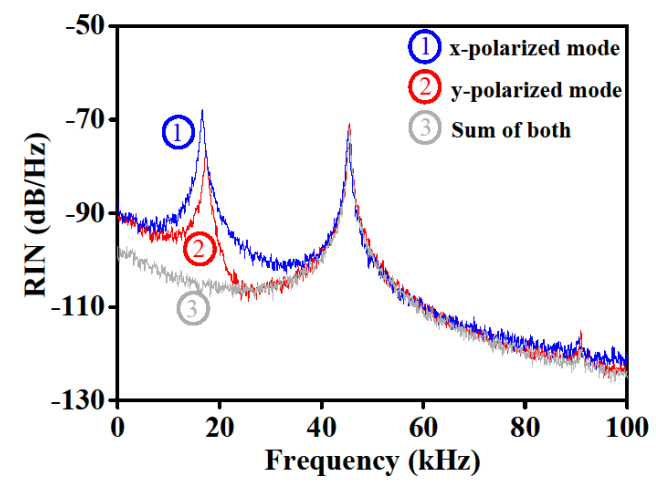

Fig. 2. Typical RIN spectra of a dual mode solid state laser recorded using an electrical spectrum analyzer (ESA) with $100 \mathrm{~Hz}$ resolution bandwidth without the nonlinear absorber into the laser.

The impact of a nonlinear absorber is studied in terms of the excess noise measured around resonance frequencies of the inphase and anti-phase frequencies. By incorporating the $100-\mu \mathrm{m}-$ thick Silicon (Si) plate in (100)-cut, intensity-dependent losses are induced in the laser cavity due to the Two-Photon Absorption (TPA) mechanism. Furthermore, this uncoated Si plate acts as an intra-cavity filter, which replaces the silica étalon.

We first consider the configuration shown in Fig. 1(a). In this first configuration, the nonlinear absorber is placed in between the birefringent crystal and the output coupler, i.e., where the two laser modes are perfectly superimposed in the cavity. Fig. 3(a) renders the recorded RIN spectra, where the in-phase peak is reduced by about $32 \mathrm{~dB}$ as compared to the laser without TPA (cf. RIN spectra depicted in Fig. 2). However, the anti-phase peak remains unchanged even though its amplitude is slightly reduced by a few $\mathrm{dB}$ compared to case without. This behaviour can be easily understood keeping in mind that the antiphase noise occurs for a constant total power. In other words, the antiphase noise does not bring any intensity fluctuations provided that the total power is measured (see Fig. 2), whereas the nonlinear losses brought by the TPA mechanism depend on the total optical power.

Although this behavior is intuitively expected, this performance observed is not straightforward. Indeed this behavior suggests that the TPA mechanism in $\mathrm{Si}$ is not sensitive to the polarization state of the interacting lightwaves but only to the total power. To go further and validate this intuitive prediction, the orientation of the 
crystallographic axes of the Si plate was checked with respect to the polarization states of the laser to observe whether it has any impact on anti-phase noise level. To this aim, the Si plate is placed on a rotating mount that enables rotation around the optical axis of the laser. Fig. 3(b) shows the evolution of the RIN level for the $x-$ polarized mode at the anti-phase frequency versus the rotation angle of the Si plate. For each orientation, the intensities of the cross-polarized modes ( $\mathrm{x}$ and $\mathrm{y}$ ) have been carefully equalized by a fine adjustment of the nonlinear plate position as reported in the inset of Fig. 3(b). These experimental results render that the orientation of the crystallographic axes of the Si plate does not play any significant role on the antiphase fluctuations. Moreover, these results suggest that the two-photon absorption mechanism in $\mathrm{Si}$ is not able to act selectively on the lightwave intensity fluctuations of each polarization state.
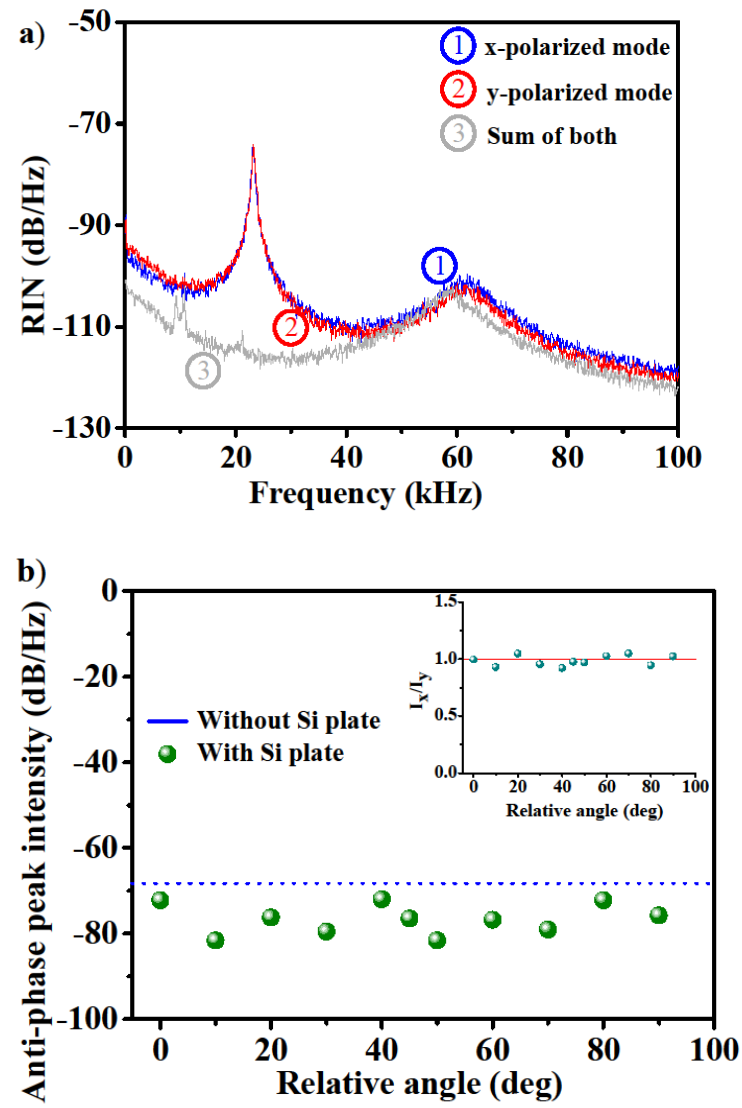

Fig. 3. (a) RIN spectra corresponding to the laser configuration shown in Fig. 1(a) (ESA resolution bandwidth of $100 \mathrm{~Hz}$ ). (b) Evolution of the RIN level at the anti-phase frequency versus the relative rotation angle between crystal axes of the $\mathrm{Si}$ plate and polarization directions is depicted in dotted line. Evolution of the RIN level at the anti-phase frequency without the Si plate into the laser is depicted in dashed line. The inset shows the evolution of the intensities ratio of two orthogonal modes versus the relative rotation angle.

Accordingly, if one targets to reduce the antiphase noise, the nonlinear absorber has to act on the two polarization modes independently. In essence, in the buffer reservoir description (see Ref. [18]), one has to introduce at least two reservoirs in order to dampen the intensity fluctuations of the two photon populations. To end up with such operating condition, we slightly lift the spatial degeneracy of the two modes in the Si plate as depicted in configuration of Fig. 1b. In practice, when the Si plate is just inserted in between the active medium and the birefringent displacer, the spatial separation hence occurs in both the Si plate and the active medium. Note that the mode coupling strength is kept constant in the active medium as compared to configuration shown in Fig. 1a, which ensures potentially the same level of antiphase noise for the two configurations depicted in Fig. 1. Moreover, it must be noted that the spatial separation being small $(20 \mu \mathrm{m})$ with respect to the mode diameter $(120 \mu \mathrm{m})$, the two modes share a large area of the nonlinear two photon absorber, while only a small part of each mode interacts with independent areas of the nonlinear absorber. As a result, the Si plate is now expected to induce at the same time common losses and selective losses for the two modes leading to a reduction of both in-phase and anti-phase noise peaks. Due to solid state laser realization based on this configuration, a $25 \mathrm{~dB}$ noise level reduction is obtained on the two peaks as depicted in Fig. 4 compared to the ones depicted in Fig. 2 and Fig. 3. These results demonstrate a detrimental role played by even a tiny spatial separation of the dual modes in the Si plate.

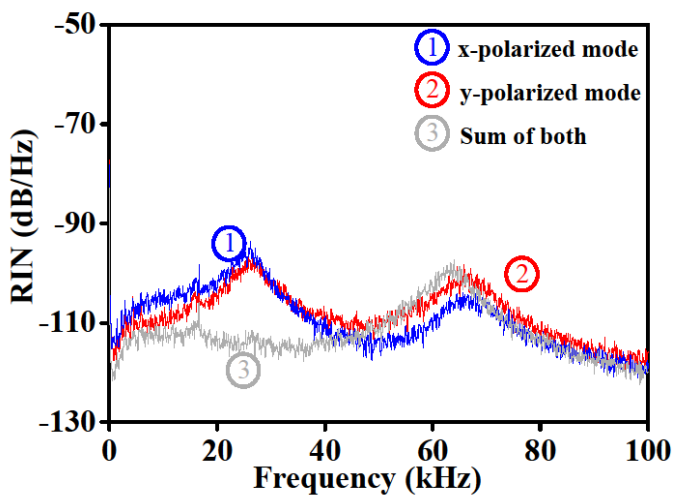

Fig. 4. RIN spectra corresponding to the laser configuration of Fig. 1(b) (ESA resolution bandwidth: $100 \mathrm{~Hz}$ ).

In summary, we have investigated and reported for the first time the impact of an intra-cavity nonlinear absorber on the noise properties of a dual-frequency solid-state laser. This study is conducted in a dual frequency Er,Yb:glass laser containing a Silicon plate which induces intensity-dependent two-photon absorption losses. In particular, we have demonstrated a reduction in the RO noise similar to the case of single frequency solid state laser, provided that this noise originates from in-phase fluctuations. Conversely, the TPA mechanism fails in damping the noise originating from antiphase fluctuations. By modifying the laser architecture so that a slight spatial separation in the nonlinear absorber is created between the two modes, we have been able to observe a simultaneous $25 \mathrm{~dB}$ reduction of both in-phase and antiphase noise spectra. This latest configuration, in which each laser mode interacts with an independent region of the nonlinear TPA, makes the anti-phase fluctuations impactful in the nonlinear absorber. 
This work opens promising perspectives in several domains, where dual-frequency lasers are employed, such as in LIDARRADAR and in the generation and optical distribution of high purity microwave signals $[5,7]$. Indeed, while in-phase noise spectra can be dampened using a large variety of techniques, the antiphase noise contributions are extremely difficult to remove. Up to date, this is the first demonstration of a dual frequency solidstate laser inherently free of both anti-phase and in-phase RIN noise spectra. Finally, this work also opens understanding of various fundamental applications where two laser beams are required and where their intensity noise spectra play a central role, such as in coherent population trapping employed in atomic clocks [23]. Future work is planned to extend the three-rateequation formalism [21] to dual-frequency lasers in order to further reduce the remaining RIN peaks tentatively up to the shot noise level.

Funding. Délégation Générale de l'Armement (DGA); Région Bretagne, France

Acknowledgment. The authors would like to acknowledge Afshin Daryoush (Drexel Univ.) for fruitful discussions and Cyril Hamel for technical support.

\section{References}

1. P. Nerin, P. Puget, P. Besesty, and G. Chartier, Electron. Lett. 33, 491 (1997).

2. W. Du, S. Zhang, and Y. Li, Opt. Laser Eng. 43, 1214 (2005).

3. L. Morvan, D. Dolfi, N. D. Lai, J. P. Huignard, M. Brunel, F. Bretenaker, and A. Le Floch, Appl. Opt. 41, 5702 (2002).

4. G. Grosskopf, D. Rohde, R. Eggemann, S. Bauer, C. Bornholdt, W. Mohrle, and B. Sartorius, IEEE Photon. Tech. Lett. 12, 1692 (2000).

5. [5] M. Alouini, B. Benazet, M. Vallet, M. Brunel, P. Di Bin, F. Bretenaker, A. Le Floch, and P. Thony, IEEE Photon. Tech. Lett. 13, 367 (2001).

6. M. Brunel, A. Amon, and M. Vallet, Opt. Lett. 30, 2418 (2005).

7. G. Pillet, L. Morvan, M. Brunel, F. Bretenaker, D. Dolfi, M. Vallet, J. P. Huignard, and A. Le Floch, J. Lightwave. Technol. 26, 2764 (2008).

8. D. E. McCumber, Phys. Rev. 141, 306 (1966).

9. F. T. Arecchi, G. L. Lippi, G. P. Puccioni, and J. R. Tredicce, Opt. Comm. 51, 308 (1984).

10. K. Otsuka, P. Mandel, S. Bielawski, D. Derozier, and P. Glorieux, Phys. Rev. A 46, 1692 (1992).

11. M. Alouini, F. Bretenaker, M. Brunel, A. Le Floch, M. Vallet, and P. Thony, Opt. Lett. 25, 896 (2000)

12. M. Alouini, M. Brunel, F. Bretenaker, M. Vallet, and A. Le Floch, IEEE Photon. Technol. Lett. 10, 1554 (1998).

13. C. Czarny, M. Alouini, C. Larat, M. Krakowski, and D. Dolfi, Electron. Lett. 40, 942 (2004).

14. L. Yifei, J. C. Amarildo, S. M. Goldwasser, and P. R. Herczfeld, IEEE Trans. Microw. Theory Tech. 49, 2048 (2001).

15. A. El Amili, G. Loas, S. De, S. Schwartz, G. Feugnet, J. P. Pocholle, F. Bretenaker, and M. Alouini, Opt. Lett. 37, 4901 (2012).

16. C. C. Harb, M. B. Gray, H.-A. Bachor, R. Shilling, P. Rottengatter, I. Freitag, and H. Welling, J. Quantum Electron. 30, 2907 (1994)

17. S. Taccheo, P. Laporta, O. Svelto, and G. de Geronimo, Opt. Lett. 21, 1747 (1996).

18. G. Baili, M. Alouini, C. Moronvalle, D. Dolfi, et F. Bretenaker, "Broad bandwidth shot-noise limited class-A operation of a monomode semiconductor fiber based ring laser" Opt. Let.t 31, 62 (2006).
19. G. Baili, L. Morvan, M. Alouini, D. Dolfi, F. Bretenaker, I. Sagnes, and A. Garnache, Opt. Lett. 34, 3421 (2009).

20. R. van Leeuwen, B. Xu, L. S. Watkins, Q. Wang, and C. Ghosh, Proc. SPIE 6975, Enabling Photonics Technologies for Defense, Security, and Aerospace Applications IV, 69750K (2008).

21. A. El Amili, G. Kervella, and M. Alouini, Opt. Express 21, 8773 (2013)

22. A. El Amili, G. Loas, L. Pouget, and M. Alouini, Opt. Lett. 37, 5014 (2014).

23. T. Zanon, S. Guérandel, E. de Clercq, D. Holleville, N. Dimarcq, and A. Clairon, Phys. Rev. Lett. 94, 193002 (2005). 


\section{References}

1. P. Nerin, P. Puget, P. Besesty, and G. Chartier, "Self-mixing using a dualpolarisation Nd:YAG microchip laser," Electron. Lett. 33, 491 (1997).

2. W. Du, S. Zhang, and Y. Li, "Principles and realization of a novel instrument for high performance displacement measurement nanometer laser ruler," Opt. Laser Eng. 43, 1214-1225 (2005).

3. L. Morvan, D. Dolfi, N. D. Lai, J. P. Huignard, M. Brunel, F. Bretenaker, and A. Le Floch, "Building blocks for a two-frequency laser lidar-radar: a preliminary study," Appl. Opt. 41, 5702-5712 (2002).

4. G. Grosskopf, D. Rohde, R. Eggemann, S. Bauer, C. Bornholdt, W. Mohrle, and B. Sartorius, "Optical millimeter-wave generation and wireless data transmission using a dual-mode laser," IEEE Photon. Tech. Lett. 12, 1692-1694 (2000).

5. M. Alouini, B. Benazet, M. Vallet, M. Brunel, P. Di Bin, F. Bretenaker, A. Le Floch, and P. Thony, "Offset phase locking of Er:Yb:Glass laser eigenstates for RF photonics applications," IEEE Photon. Tech. Lett. 13, 367-369 (2001).

6. M. Brunel, A. Amon, and M. Vallet, "Dual-polarization microchip laser at $1.53 \mu \mathrm{m}$," Opt. Lett. 30, 2418-2420 (2005).

7. G. Pillet, L. Morvan, M. Brunel, F. Bretenaker, D. Dolfi, M. Vallet, J. P. Huignard, and A. Le Floch, "Dual-frequency laser at $1.5 \mu \mathrm{m}$ for optical distribution and generation of high-purity microwave signals," J. Lightwave. Technol. 26, 2764-2773 (2008).

8. D. E. McCumber, "Intensity Fluctuations in the Output of $\mathrm{cw}$ Laser Oscillators. I," Phys. Rev. 141, 306-322 (1966).

9. F.T. Arecchi, G.L. Lippi, G.P. Puccioni, and J.R. Tredicce, "Deterministic chaos in lasers with injected signal," Opt. Comm. 51, pp. 308-314 (1984).

10. K. Otsuka, P. Mandel, S. Bielawski, D. Derozier, and P. Glorieux, "Alternate time scales in multimode lasers," Phys. Rev. A 46, 1692-1695 (1992).

11. M. Alouini, F. Bretenaker, M. Brunel, A. Le Floch, M. Vallet, and P. Thony, "Existence of two coupling constants in microchip lasers," Opt. Lett. 25, 896-898 (2000).

12. M. Alouini, M. Brunel, F. Bretenaker, M. Vallet, and A. Le Floch, "Dual tunable wavelength $\mathrm{Er}, \mathrm{Yb}$ :glass laser for terahertz beat frequency generation," IEEE Photon. Technol. Lett. 10, 1554-1556 (1998).

13. C. Czarny, M. Alouini, C. Larat, M. Krakowski, and D. Dolfi, "THz-dualfrequency $\mathrm{Yb3}+\mathrm{KGd}(\mathrm{WO} 4) 2$ laser for continuous wave $\mathrm{THz}$ generation through photomixing," Electron. Lett. 40, 942-943 (2004).

14. L. Yifei, J. C. Amarildo, S. M. Goldwasser, and P. R. Herczfeld, "Rapidly Tunable Millimeter-Wave Optical Transmitter for Lidar-Radar," IEEE Trans. Microw. Theory Tech. 49, 2048-2054 (2001).

15. A. El Amili, G. Loas, S. De, S. Schwartz, G. Feugnet, J. P. Pocholle, F. Bretenaker, and M. Alouini, "Experimental demonstration of a dualfrequency laser free from antiphase noise, " Opt. Lett. 37, 4901-4903 (2012).

16. C. C. Harb, M. B. Gray, H.-A. Bachor, R. Shilling, P. Rottengatter, I. Freitag, and H. Welling, "Suppression of the intensity noise in a diodepumped neodymium:YAG nonlinear ring laser," J. Quantum Electron. 30, 2907-2913 (1994)

17. S. Taccheo, P. Laporta, O. Svelto, and G. de Geronimo, "Intensity noise reduction in a single-frequency ytterbium-codoped erbium laser," Opt. Lett. 21, 1747-1749 (1996).

18. G. Baili, M. Alouini, C. Moronvalle, D. Dolfi, et F. Bretenaker, "Broad bandwidth shot-noise limited class-A operation of a monomode semiconductor fiber based ring laser" Opt. Let.t 31, 62 (2006).

19. G. Baili, L. Morvan, M. Alouini, D. Dolfi, F. Bretenaker, I. Sagnes, and A. Garnache, "Experimental demonstration of a tunable dual-frequency semiconductor laser free of relaxation oscillations," Opt. Lett. 34, 34213423 (2009).
20. R. van Leeuwen, B. Xu, L. S. Watkins, Q. Wang, and C. Ghosh, "Low noise high power ultra-stable diode pumped $\mathrm{Er}-\mathrm{Yb}$ phosphate glass laser," Proc. SPIE 6975, Enabling Photonics Technologies for Defense, Security, and Aerospace Applications IV, 69750K (2008).

21. A. El Amili, G. Kervella, and M. Alouini, "Experimental evidence and theoretical modeling of two-photon absorption dynamics in the reduction of intensity noise of solid-state Er:Yb lasers," Opt. Express 21, 8773-8780 (2013)

22. A. El Amili, G. Loas, L. Pouget, and M. Alouini, "Buffer reservoir approach for cancellation of laser resonant noises," Opt. Lett. 37, 5014 5017 (2014)

23. T. Zanon, S. Guérandel, E. de Clercq, D. Holleville, N. Dimarcq, and A. Clairon, "High Contrast Ramsey Fringes with Coherent-PopulationTrapping Pulses in a Double Lambda Atomic System, " Phys. Rev. Lett. 94, 193002 (2005). 